\title{
Aktuelle Themen
}

Standpunkte: „Ziemlich beste Freunde“ - Innovative

Kooperationsmodelle mit Wettbewerbern im Zeitalter der Digitalisierung

06

Bayerischer Rundfunk und MedienWirtschaft:

Diskussionsbeiträge zum Forum Medienmanagement auf den Medientagen München 2018

\section{Aufsätze}

Social Media: Institutionalisierungsprozesse

als interdisziplinäre Forschungsaufgabe

Prof. em. Dr. Otfried Jarren, Universität Zürich, Schweiz

Zusammenarbeit von Mensch und Technik im Medienbereich

Prof. Dr. phil. Dr.-Ing. Birgit Spanner-Ulmer, Bayerischer Rundfunk

Prof. Dr.-Ing. Ralph Bruder, Technische Universität Darmstadt

Rechtliche Antworten auf die Künstliche Intelligenz im Mediensektor

32

Priv.-Doz. Dr. Jan Oster, LL.M., Universität Leiden, Niederlande

\section{Service}

Deutsche Gesellschaft für Publizistik- und

Kommunikationswissenschaft (DGPuK)

Zwei Call for Papers: „Innovation in der Medienproduktion und -distribution“,

„Workshop für den wissenschaftlichen Nachwuchs"

Kongresse - Tagungen - Events

Janis Brinkmann: Verlagspolitik in der Zeitungskrise I Prof. Dr. Thomas Breyer-Mayländer, Hochschule Offenburg

Christiana Gransow: Public Value-Konzepte im öffentlichen Rundfunk I

Prof. Dr. Hardy Gundlach, Hochschule für Angewandte Wissenschaften Hamburg

Kim Otto/Andreas Köhler (Hrsg.): Crossmedialität im Journalismus und in der Unternehmenskommunikation I

Prof. Dr. Wolfgang Fuchs, Hochschule der Medien Stuttgart

Catrin Misselhorn: Grundfragen der Maschinenethik, 3. Auflage I

Prof. Dr. Sarah Spitzer, Hochschule der Medien Stuttgart

Call for Participation 